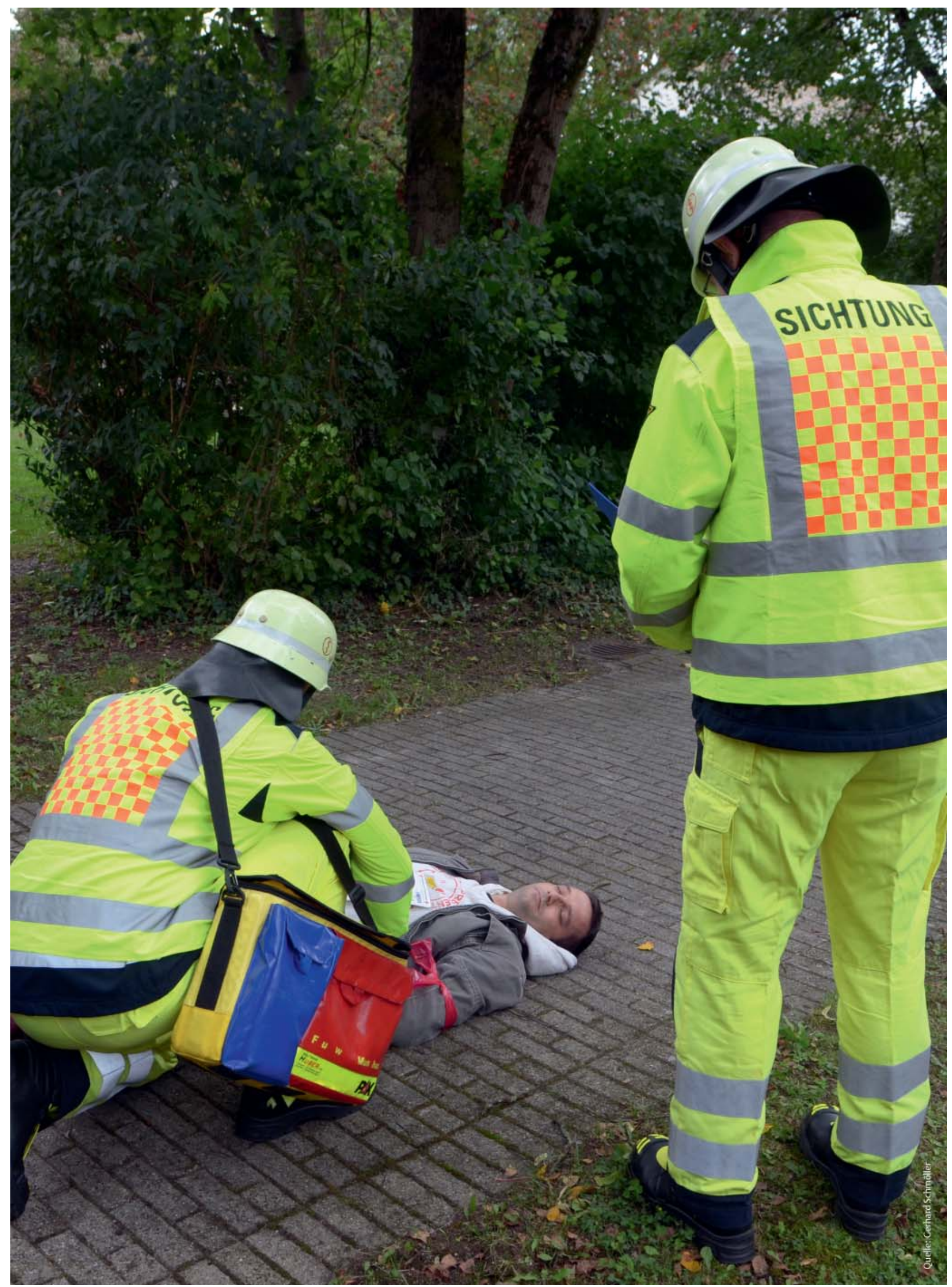




\section{Sichtung und Vorsichtung bei Massenanfall von Verletzten und Erkrankten}

Gerhard Schmöller, Fritz Hagen

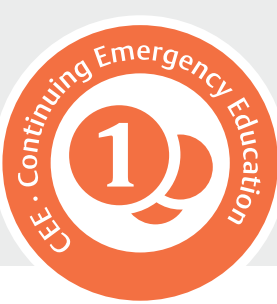

Laut Definition der Bundesärztekammer (BÄK) ist die Sichtung eine ärztliche Aufgabe. Leider sind selbst im Regelrettungsdiensteinsatz Ärzte und Notärzte nicht immer als erste an der Einsatzstelle - und schon gar nicht beim Massenanfall von Verletzten und Erkrankten (MANV/MANE). Somit müssen auch Nichtärzte die Sichtung durchführen. Um diesem Umstand Rechnung tragen zu können, wurde in Bayern der Begriff „(nichtärztliche) Vorsichtung“ gewählt [1].

\section{Nichtärzte übernehmen initiale Sichtung}

Zur WM 2006 waren Vorbereitungen der nichtpolizeilichen Gefahrenabwehrsysteme nötig. Sie haben deutliche Verbesserungen der Konzepte und Maßnahmen bei Massenanfall von Verletzten und Erkrankten (MANV/MANE) möglich gemacht. Ergebnisorientiert zusammenarbeiten zu müssen, eingebettet in durch den Spielplan festgelegte Termine, verlieh den Arbeitsgruppen Rückenwind. Davon profitiert das System in ganz Deutschland bis heute.

Anhand der Vorgaben der FIFA und der Bundesregierung wurden Szenarien mit 200 bis 300 Patienten geplant: Es zeichnete sich schnell ab, dass eine Sichtung durch Notärzte bzw. den Leitenden Notarzt (LNA) zu einer Zeitverzögerung führen würde, die nicht hinnehmbar wäre. Deshalb erschien es notwendig, andere Wege zu gehen.

Bereits 2004 wurden Versuche durchgeführt, die untersuchten, inwieweit Nichtärzte die Aufgabe der initialen Sichtung übernehmen können [2]. Seitdem ist dieses Verfahren im Rettungsdienstbereich München etabliert. Zuletzt nahmen Rettungsassistenten der Münchner Berufsfeuerwehr die Vorsichtung wahr, als am 22.07.2016 ein 18-Jähriger in München Amok lief.

Bereits im Rahmen der 4. Sichtungs-Konsensus-Konferenz der Schutzkommission 2012 entdeckten die Experten unter anderem, dass mehrere Sichtungskonzepte existieren. Es konnte sich allerdings kein universell anwendbares Konzept durchsetzen.

In Bayern erteilte das Bayerische Staatsministerium des Innern, für Bau und Verkehr (BayStMI) im November 2013 den Auftrag, „auf der Grundlage des mSTaRT-Algorithmus ein fachlich an internationalen Standards ausgerichtetes Modell für Bayern zu entwickeln [...]“, um dieses in die neu zu erstellende Richtlinie zur Bewältigung von
Ereignissen mit einem Massenanfall von Notfallpatienten und Betroffenen (MAN-RL) [1] integrieren zu können [4]. Der Algorithmus ist unter folgendem Link online abrufbar: https://eref.thieme.de/W7HZW.

\section{Integration der Vorsichtung in den MANV-Einsatz}

Der Rettungsassistent bzw. Notfallsanitäter des Rettungswagens (RTW), der zuerst eintrifft, soll die vorläufige Einsatzführung übernehmen [1] und erste wichtige Maßnahmen ergreifen wie:

- Bestätigung der Funktionsübernahme und Rückmeldung der „Lage auf Sicht“

- Kennzeichnung seiner Person (z. B. mit Weste „1. RTW“)

- Erkundung (inkl. einer Gefahrenanalyse) und Lagemeldung

- Abstimmung mit anderen Fachdiensten

- Organisation der medizinischen Erstversorgung in enger Abstimmung mit dem ersteintreffenden Notarzt (gekennzeichnet durch die Weste „1. NA“)

- dabei muss er weiterhin die Vorsichtung organisieren (Die Vorsichtung selbst wird durch den RTW durchgeführt, der als Nächster eintrifft).

\section{Lagemeldung}

Je früher die Zahl der Verletzten und die jeweilige Verletzungsart gemeldet werden, desto schneller kann die Leitstelle die notwendigen Ressourcen für Behandlung und Transport anfordern; auch können die klinischen Versorgungsstätten informiert werden und Kapazitäten bereitstellen.

Selbst bei zeitgleichem Einsatz mehrerer Sichtungsteams wird es dauern, bis das Sichtungsergebnis feststeht. 
Merke

Für einen allerersten Überblick ist es gut, die liegenden (immobilen) Verletzten zu zählen und die Zahl der Gehfähigen zu schätzen.

Mit diesen Zahlen zeichnet sich die Größenordnung des Einsatzes effektiv ab. Die Leitstelle kann anhand dieser Daten sehr früh entscheiden, ob die Klinikkapazitäten des eigenen Organisationsbereichs [5] ausreichen oder Kliniken anderer Leitstellenbereiche belegt werden müssen. Damit geht auch die Alarmierung von Behandlungsund Transportkapazitäten einschließlich der Luftrettungsmittel einher.

\section{Gehfähige}

Häufig ist in diesem Zusammenhang die Rede von Leichtverletzten oder Betroffenen. Diese Klassifizierung setzt jedoch voraus, dass die Gehfähigen bereits gesichtet wurden. In der frühen Phase des Einsatzes sind in der Regel dafür keine Ressourcen vorhanden. Die Gehfähigen sollten sehr zeitig zu einem Sammelplatz geschickt werden; dort können die Helfer später die Differenzierung in Betroffene und Verletzte vornehmen.

Merke

Als Betroffene gelten Personen, die durch ein Schadensereignis bedroht oder geschädigt wurden, ohne verletzt zu sein [6].

\section{Vorsichtung}

\section{Ausstattung/Ausrüstung}

Die notwendige Ausstattung ist nach einsatztaktischen Gesichtspunkten vorzuhalten - einschließlich der Kennzeichnung des Vorsichtungsteams (Westen). Denkbar ist die Vorhaltung auf Regel-RTW, speziellen SichtungsRTW, Feuerwehrfahrzeugen oder die Vorhaltung bei Sanitätsdiensten.

\section{Aus- und Fortbildung}

Unstrittig ist, dass das Sichtungsteam eine Aus- und Fortbildung benötigt. Bayern empfiehlt eine Ausbildung durch zertifizierte Multiplikatoren, die 4 Unterrichtseinheiten (UE) umfasst [7]. Auch zum Kompetenzerhalt gibt es Empfehlungen: Allerdings zeigte sich bereits nach einem Jahr ein Kompetenzverlust [8] - weshalb diese Empfehlung kritisch zu hinterfragen ist.

Das anfängliche Konzept zum Erhalt der Kompetenz der Berufsfeuerwehr München sah eine jährlich zu bearbeitende Computersimulation vor; diese schaffte allerdings nur eine Kompetenzillusion. Geht es um ernsthaften Kompetenzerhalt ist eine praktische Simulation unerlässlich - aus Sicht des Verfassers sollte diese mindestens einmal jährlich durchgeführt werden und auch die Begleitfaktoren aufgreifen.

\section{Begleitfaktoren bei der Vorsichtung}

Bestandteil der Ausbildung sollte sein, die potenziellen Sichtungsteams auf erschwerende Aspekte vorzubereiten:

- Retter sind selten mit der Aufgabe Vorsichtung konfrontiert; allein daraus resultiert eine gewisse Unsicherheit.

- Die Konfrontation mit sehr vielen Schwerverletzten und -erkrankten erhöht die emotionale Belastung.

- Die sehr kurze Kontaktzeit zum jeweiligen Patienten ist, im Unterschied zum Tagesgeschäft, mehr als ungewohnt; ein Gefühl der Hilflosigkeit entsteht.

- Die Erstretter müssen die Gehfähigen anweisen, sich zu einer Sammelstelle zu begeben; das bindet eigene Ressourcen und kostet Zeit.

- Ersthelfer haben die Erwartung, dass sie mit Eintreffen des Vorsichtungsteams von ihrer Aufgabe entbunden sind, müssen jedoch weiter beim Verletzten bleiben; auch das ist für die Kommunikation belastend.

- Angehörige oder Dritte halten das Team fest und drohen Gewalt an, wenn der Retter einen Verletzten verlassen muss. Diese Erfahrung musste das Sichtungsteam beim Einsatz im Rahmen des Amoklaufs in München am 22.07.2016 machen.

\section{Organisation der Vorsichtung}

Das erste Sichtungsteam wird dort eingesetzt, wo die „potenziell am schwersten Verletzten“ zu erwarten sind - also am Schwerpunkt des Ereignisses. Sofern mehrere Teams eingesetzt werden sollen, sind die einzelnen Abschnitte eindeutig zu kennzeichnen und den Teams eindeutig zuzuweisen.

\section{Durchführung der Vorsichtung}

Das Sichtungsteam besteht aus 2 Personen. Es bleibt zusammen - das ist psychologisch sinnvoll - und arbeitet sich gemeinsam von Patient zu Patient vor. Ein Teammitglied liest dabei die Fragen des Algorithmus vor (siehe https://eref.thieme.de/W7HZW) und dokumentiert ( $\vee$ Abb. 1). Das andere Teammitglied untersucht und interveniert (Freimachen des Atemwegs oder Blutstillung).

\footnotetext{
Merke

Das Sichtungsteam muss unbedingt darauf achten, dass jeder Liegende vorgesichtet wird.
}

Eine eindeutige und erkennbare Kennzeichnung verhindert eine Mehrfachsichtung in der Erstphase. In München wird dazu eine Verletztenanhängekarte (VAK) mit der jeweiligen Sichtungskategorie (SK) farblich gekennzeichnet ( $\triangleright$ Tab. 1). Die Retter können diese Farbkennzeich- 


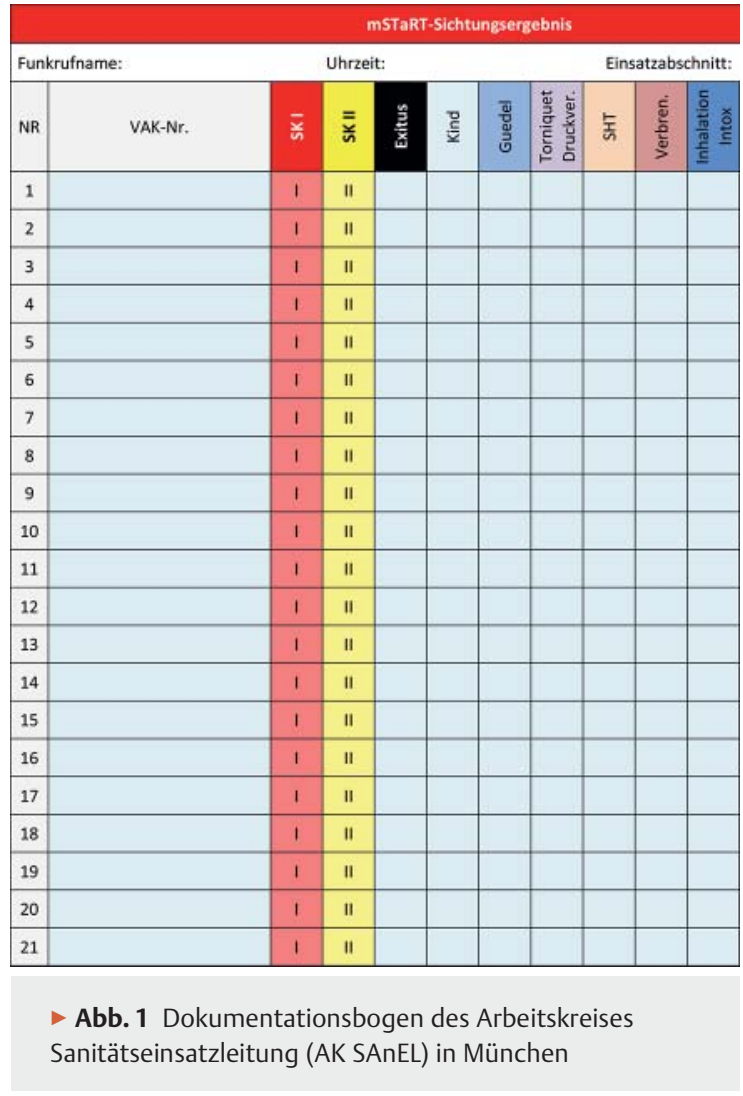

- Tab. 1 Sichtungskategorien und Farben [3].

\begin{tabular}{|l|l|l|}
\hline $\begin{array}{l}\text { Sichtungs- } \\
\text { kategorie (SK) }\end{array}$ & Beschreibung & Konsequenz \\
\hline $\begin{array}{l}\text { I } \\
\text { (rot) }\end{array}$ & vital bedroht & Sofortbehandlung \\
\hline $\begin{array}{l}\text { II } \\
\text { (gelb) }\end{array}$ & $\begin{array}{l}\text { schwer verletzt/ } \\
\text { erkrankt }\end{array}$ & $\begin{array}{l}\text { dringliche } \\
\text { Behandlung }\end{array}$ \\
\hline $\begin{array}{l}\text { III } \\
\text { (grün) }\end{array}$ & $\begin{array}{l}\text { leicht verletzt/ } \\
\text { erkrankt }\end{array}$ & $\begin{array}{l}\text { nicht dringliche } \\
\text { Behandlung }\end{array}$ \\
\hline $\begin{array}{l}\text { IV } \\
\text { (blau) }\end{array}$ & $\begin{array}{l}\text { ohne } \\
\text { Überlebenschance }\end{array}$ & $\begin{array}{l}\text { palliative } \\
\text { Versorgung }\end{array}$ \\
\hline $\begin{array}{l}\text { EX } \\
\text { (schwarz) }\end{array}$ & Tote & \\
\hline
\end{tabular}

nung austauschen, das ermöglicht eine schnelle Umkategorisierung - falls nötig. In der Münchner Vorsichtungstasche sind nur VAK mit gelben und roten Farbkennzeichnungen vorgesteckt, da bei den Liegenden nur diese beiden Sichtungskategorien zu erwarten sind.

Die Sichtung soll hier exemplarisch am Beispiel des „Algorithmus - nichtärztliche Vorsichtung ,Modell Bayern'“ dargestellt werden; es wurde vom „Ausschuss Ärztlicher Leiter Rettungsdienst Bayern“ erstellt.
- Gehfähige Personen werden angewiesen, eine Sammelstelle aufzusuchen. Diese Stelle wurde zuvor als räumlicher Einsatzabschnitt vom Einsatzleiter definiert, und dem Sichtungsteam/den Sichtungsteams wurde dessen Lage mitgeteilt. Zur Aus- und Fortbildung der Ersthelfer gehört auch, die kurze Kommunikation mit den Gehfähigen zu üben, um sich nicht in Diskussionen verwickeln zu lassen, was den Sichtungsvorgang verzögern würde.

- Werden bei einem Patienten Verletzungen festgestellt, die nicht mit dem Leben vereinbar sind, wird dieser nur gekennzeichnet und in die Sichtungsdokumentation aufgenommen. Somit ist für die Einsatzleitung erkennbar, dass sie einen Arzt bitten muss, den Tod festzustellen.

- Für die weitere Einschätzung müssen die Helfer nach dem ABCDE-Schema vorgehen.

- Für die Vergabe der Sichtungskategorie I „Sofortbehandlung“ reicht es, wenn eine der folgenden Fragen negativ beantwortet wird.

- Wie aus dem Algorithmus ersichtlich, sind dies die Fragen „Atmung vorhanden“, „Atemfrequenz“, „Spritzende Blutung“, „Radialispuls vorhanden“, „Befolgen einfacher Befehle“ und „Inhalationstrauma mit Stridor".

- Die weiteren Fragen müssen nicht mehr geprüft werden. Es werden nur die Maßnahmen „Freimachen des Atemwegs“ und „Blutstillung“ durchgeführt.

- Werden die Fragen so beantwortet, dass sie in den positiveren Bereich fallen, ist der liegende Patient mit der Sichtungskategorie II „Dringende Behandlung“ zu kennzeichnen. Auch dieser ist in der Sichtungsliste zu dokumentieren. ( $\triangleright$ Abb. 1)

\section{Kennzeichnung von Patienten}

Die Branddirektion München hat eine Handlungsanweisung „Vorsichtung“ erstellt. Die Fassung vom 01.12.2015 legt fest, dass Patienten mit der Sichtungskategorie I mit der rot gesteckten VAK sowie einer roten Flatterleine zu kennzeichnen sind, Patienten mit SK II mit einer gelb gesteckten VAK ( $\triangleright$ Abb. 2). Patienten mit einer tödlichen Verletzung werden nur mit einer schwarz-gelben Flatterleine gekennzeichnet.

\section{Algorithmus mit Frage nach ABC-Lage}

Der mSTaRT-Vorsichtungsalgorithmus München unterscheidet sich im Wesentlichen vom Algorithmus Bayern dadurch, dass der Frage „Patient gehfähig?“ noch die Frage „ABC-Lage?“ vorgeschaltet ist ( $>$ Abb. 3). Dabei gilt es zwei Aspekte zu beachten: 
- Tab. 2 Geruch und Substanz [9].

\begin{tabular}{|l|l|}
\hline Geruch nach & Substanz \\
\hline Knoblauch & Hautkampfstoffe wie z. B. Schwefellost \\
\hline Senf & Hautkampfstoffe wie z. B. Senfgas \\
\hline Fisch & Hautkampfstoffe wie z. B. Stickstofflost und Lewisit \\
\hline Heu & Lungenkampfstoff Phosgen \\
\hline Bittermandel & \begin{tabular}{l} 
Blutkampfstoffe wie z. B. Arsenwasserstoff, \\
Cyanwasserstoff und Chlorcyan \\
\hline Chlor
\end{tabular} \\
\hline
\end{tabular}

- Tab. 3 mSTaRT-Algorithmus: unterschiedliche Fragen beim Kind und beim Erwachsenen.

\begin{tabular}{|l|l|}
\hline Erwachsener & Kind \\
\hline $\begin{array}{l}\text { Atemfrequenz zwischen 10 und } \\
\text { 30/min? }\end{array}$ & $\begin{array}{l}\text { Atemfrequenz zwischen } 10 \text { und } \\
40 / \text { min? }\end{array}$ \\
\hline Radialispuls vorhanden? & Rekap-Zeit Stirn kleiner 3 s? \\
\hline Kann einfache Befehle befolgen? & Reagiert adäquat? (ab 3 Jahre) \\
\hline
\end{tabular}

- entweder die Wahrnehmung von besonderen Gerüchen ( $\triangleright$ Tab. 2) und/oder

- den Umstand, dass bei mehr als einer Person gleiche Vergiftungssymptome zu beobachten sind.

\section{Was passiert mit den Gehfähigen?}

In der Erstphase des Einsatzes ist es oft schwierig einzuschätzen, ob Gehfähige verletzt oder nur betroffen sind. Dieser Nachweis lässt sich initial unter Zeitdruck nicht sinnvoll führen, ohne die originäre Aufgabe Vorsichtung zu verzögern.

\author{
Merke \\ Es ist hilfreich, einen Sammelplatz zu definieren, \\ um den Gehfähigen ein Ziel anzubieten.
}

Beim Zugunfall in Bad Aibling wurden die Gehfähigen angewiesen, sich selbst am Bahndamm in Richtung Osten zu begeben. An dieser Sammelstelle sollte nach Eintreffen geeigneten Personals die Differenzierung zwischen Betroffenen und verletzt Gehfähigen durchgeführt werden. Die gehfähigen Verletzten wurden nachgesichtet und medizinisch versorgt.

In München wird dazu der Algorithmus „Nachsichtung“ verwendet ( $\triangleright$ Abb. 4). Sofern kein kritischer Befund erhoben wurde, wird der Patient mit einer Verletztenanhängekarte mit der Sichtungskategorie III gekennzeichnet.

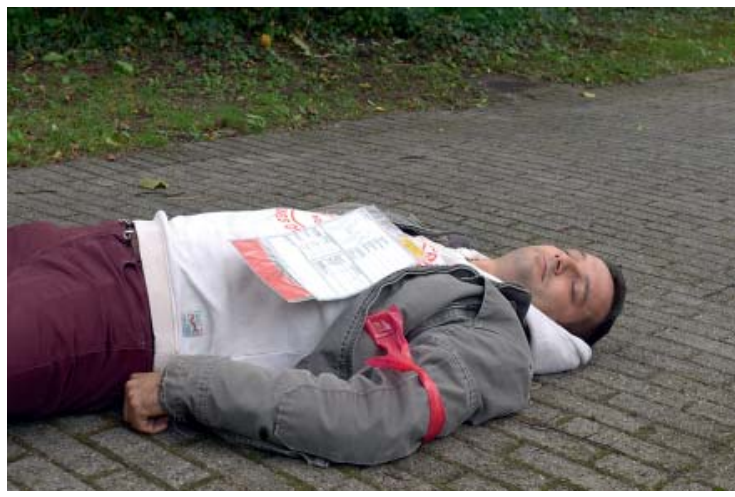

Abb. 2 Patient mit roter Flatterleine am Oberarm.

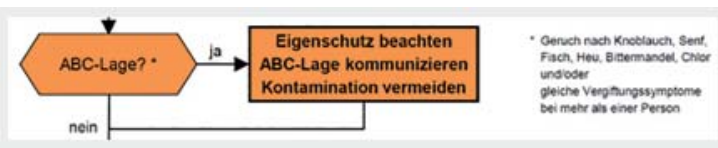

Abb. 3 ABC-Lage.

\begin{abstract}
Merke
Alle Verletzten müssen nachgesichtet werden,
\end{abstract} um kritische Befunde zu entdecken.

Bei der MANV-Übung im Oktober 2005 in München haben die Nachsichtungsteams bei den Leichtverletzten per Augenschein eine Vorauswahl getroffen. Aus diesem Grund wurden nicht alle Patienten nachgesichtet und somit auch nicht nach kritischen Befunden untersucht. In der Konsequenz konnten deshalb nicht alle kritischen Befunde identifiziert werden. Wird ein kritischer Befund erhoben, ändert sich die Sichtungskategorie mindestens in die Sichtungskategorie II oder ggf. in Sichtungskategorie I.

\section{Unterschiede bei der Vorsichtung zwischen Erwachsenem und Kind}

Eine wichtige Frage ist, ab welchem Alter die Helfer den Erwachsenen-Algorithmus anwenden müssen und bis zu welchem Alter den Kinder-Algorithmus. Im Münchner mSTaRT-Algorithmus „Vorsichtung Kind“ heißt es dazu: „abhängig von der individuellen körperlichen Entwicklung (bis zum Beginn der Pubertät; 10 - 12 Jahre)“. Bei Kindern werden z. T. andere Fragen gestellt ( $\triangleright$ Tab. 3 ).

Auch bei der Suche nach kritischen Befunden im Rahmen der Nachsichtung gehfähiger Patienten sind folgende Parameter bei Kindern angepasst.

- B-Atmung (Atemfrequenz größer als 40/min)

- C-Kreislauf (zentrale Rekap-Zeit an Stirn oder Sternum größer 3 s, HF größer 140/min, HF kleiner 80/min)

- D-Bewusstsein (AVPU schlechter als „A“)

- D-Verdacht SHT (Pupillendifferenz) 


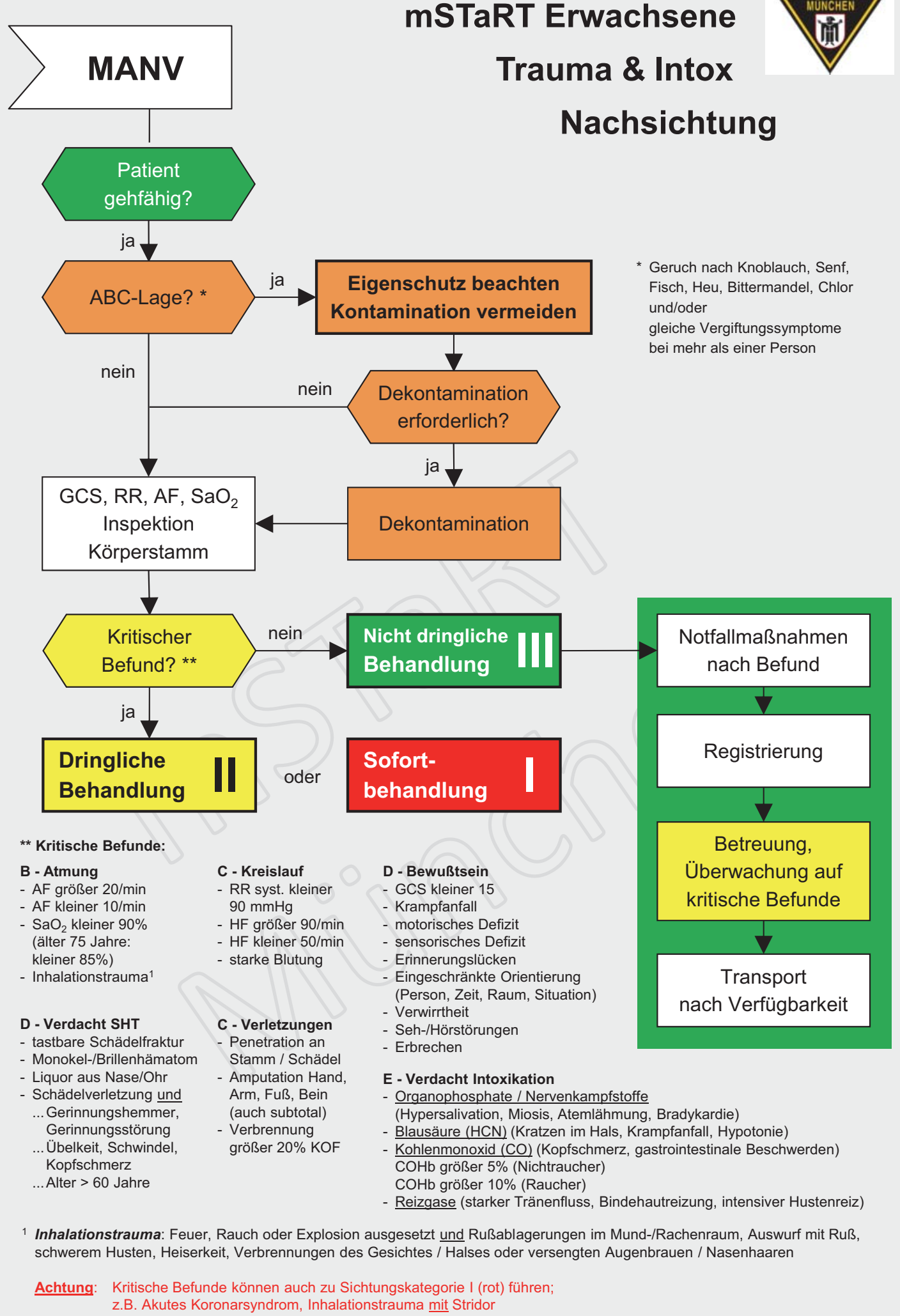

Dieser Algorithmus ist Teil der Handlungsanweisung „Nachsichtung im MANV-Einsatz“

(c) Berufsfeuerwehr München - Einsatzplanung. Version 3.5. Stand: 08.12.2015

- Abb. 4 Algorithmus zur Nachsichtung. 


\section{Dokumentation}

Wie in - Abb. 1 dargestellt, dokumentiert das Sichtungsteam auf dem dafür vorgesehenen Bogen. Bei Patienten der SK I und SK II werden die Nummern der VAK in den Bogen übernommen. Bei Patienten mit tödlicher Verletzung kann keine VAK-Nummer eingetragen werden, da diese nicht mit einer VAK gekennzeichnet werden. In den Spalten rechts neben den Sichtungskategorien sind, wenn zutreffend, die Zellen „Kind“, „Guedel“, „Tourniquet-Druckverband“, „SHT“, „Verbrennung“ und/ oder „Inhalation-Intox“ anzukreuzen. Zum Umgang mit diesem Sichtungsergebnis ist in der oben genannten Münchner Handlungsanweisung „Vorsichtung“ festgelegt, dass die Helfer das Sichtungsergebnis an den Abschnittsleiter melden müssen. Dieser meldet es dann dem Abschnittsarzt sowie der Sanitätseinsatzleitung.

\section{Mittlerweile Standard in Bayern}

Durch die im Dezember 2016 veröffentlichte MAN-RL ist die nichtärztliche Vorsichtung in Bayern mittlerweile Standard beim Massenanfall von Verletzten. Selbstverständlich sei an dieser Stelle darauf hingewiesen, dass die Vorsichtung nur eine Momentaufnahme darstellt.

\section{ACHTUNG}

Der Zustand des Patienten kann sich jederzeit ändern, die Behandlungs- und Transportteams müssen wachsam bleiben, um Veränderungen zu erkennen und entsprechend zu reagieren.

In diesem Zusammenhang ist von Einzelfällen berichtet worden, in denen sich als SK II gesichtete Patienten selbst hochkategorisiert haben, um so schnell wie möglich abtransportiert zu werden. Der Algorithmus „Nachsichtung“ bei gehfähigen Verletzten ist darüber hinaus eine Möglichkeit, kritische Befunde auch bei Leichtverletzten standardisiert und sicher zu erkennen.

\section{Vorsichtung im Regelrettungsdienst- einsatz, also Vorsichtung ToGo}

\section{FALLBEISPIEL}

Stellen Sie sich vor, Sie und Ihr Kollege sind mit Ihrem RTW zu einem Verkehrsunfall mit mehreren Verletzten alarmiert. Sie wissen, das nächste Fahrzeug erreicht erst 10 Minuten nach Ihnen den Einsatzort. Bei Eintreffen stellen Sie fest, ein PKW ist gegen einen Baum geprallt und steht fünf Meter vom
Baum entfernt in einer Wiese. Sie sehen, dass es sich um mindestens vier Patienten handelt, wovon sich zwei auf Fahrer- und Beifahrersitz im stark deformierten Fahrzeug befinden, einer sitzt betreut durch einen Ersthelfer am Fahrbahnrand, einer liegt zehn Meter vom Fahrzeug entfernt in der Wiese.

Anhand welcher Kriterien schaffen Sie sich jetzt einen Überblick? Und wie teilen Sie Ihre Ressourcen ein? Wen behandeln Sie in welchem Umfang zuerst?

Mit diesem Szenario werden Sie wahrscheinlich eher konfrontiert, als mit einem spektakulären MANV-Einsatz. Die Notwendigkeit, bei einem Einsatz begrenzte Mittel zielgerichtet einzusetzen, setzt gewisse Überlegungen und Strategien voraus. Die Sichtung von Patienten ist eine dieser Strategien, die die zielgerichtete Identifikation und damit priorisierte Versorgung von Patienten ermöglichen kann.

Sie könnten anhand der Fragen „Atmung vorhanden?“, „Atemfrequenz zwischen 10 und 30/min?", „Radialispuls vorhanden?“ und „Kann einfache Befehle befolgen?“ sehr schnell Patienten der SK I identifizieren - und somit gezielter Ihre Arbeitskraft und die Ihrer Kollegen einsetzen.

\section{KERNAUSSAGEN}

- Sichtung ist eine ärztliche Aufgabe, jedoch stehen Ärzte und Notärzte nicht immer sofort zur Verfügung, weshalb die Vorsichtung auch durch Nichtärzte durchgeführt werden muss.

- Die Besatzung des ersteintreffenden RTW sollte die vorläufige Einsatzführung übernehmen.

- Durch eine frühe Meldung der Lage, die die Zahl der liegenden und gehfähigen Patienten enthält, ist die Leitstelle in der Lage, eine angepasste Nachalarmierung bzw. Information über Aufnahmekapazitäten durchzuführen.

- Gehfähige sollen zu einem Sammelplatz geschickt werden; die Differenzierung in Leichtverletzte und Betroffene kann dort vorgenommen werden.

- Sichtung muss intensiv und praxisnah trainiert werden.

- Sichtungsalgorithmen können auch Hinweise zur Erkennung einer $A B C$-Lage enthalten.

- Im Regelrettungsdiensteinsatz können die Sichtungskriterien sehr effektiv zur schnellen Einschätzung der Verletzungsschwere eingesetzt werden.

- Die Sichtung ist sehr belastend, die Betreuung der Sichtungsteams durch Kräfte der Psychosozialen Notfallversorgung (PSNV-E) ist sicherzustellen. 


\section{Interessenkonflikt}

Die Autoren geben an, dass kein Interessenkonflikt besteht.

\section{Autorinnen/Autoren}

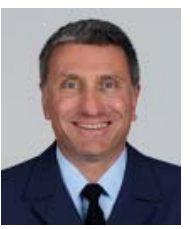

\section{Gerhard Schmöller}

leitet die Berufsfachschule für Notfallsanitäter der Landeshauptstadt München der Feuerwehrund Rettungsdienstschule München und ist Notfallsanitäter und als Organisatorischer Leiter und Einsatzleiter Rettungsdienst in Stadt und Landkreis München tätig.

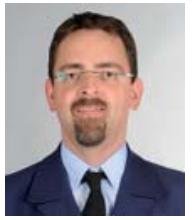

\section{Dr. Fritz Hagen}

ist Facharzt für Anästhesie und ärztliche Lehrkraft an der Berufsfachschule für Notfallsanitäter der Landeshauptstadt München der Feuerwehr- und Rettungsdienstschule München und nimmt am gemeinsamen Notarztdienst der Landeshauptstadt München, des Landkreises München und der ADAC Luftrettung (Standort Christoph 1) teil.

\section{Korrespondenzadresse}

\section{Gerhard Schmöller}

Berufsfeuerwehr München, Trainingszentrum für Rettungsmedizin

Bassermannstraße 20,

81245 München

gerhard.schmoeller@muenchen.de

\section{Literatur}

[1] Bayer. Staatsministerium des Innern, für Bau und Verkehr. Richtlinie zur Bewältigung von Ereignissen mit einem Massenanfall von Notfallpatienten und Betroffenen (MAN-RL) (06.12.2016).

[2] Gutsch W, Huppertz T, Kanz KG. Initiale Sichtung durch Rettungsassistenten. Notfall Rettungsmed 2006; 9: 384-388

[3] Protokoll der Sechsten Sichtungs-Konsensus-Konferenz der Schutzkommission in Ahrweiler am 23./24.11.2016. Im Internet: www.bbk.bund.de/DE/AufgabenundAusstattung/ Schutz_der_Gesundheit/Sichtung/sichtung_node.html Tag des Abrufs: 07.11.2017

[4] ÄLRD-Ausschuss Bayern, AG Massenanfall. Nichtärztliche Vorsichtung - Algorithmus für den Massenanfall verletzter oder chemisch intoxikierter Patienten ab dem Schulalter. http://www.aelrd-bayern.de/images/stories/pdf/Empfehlung_AG_Massenanfall_Vorsichtung_1.1.pdf Stand 20.10.2017

[5] Adams HA, Krettek C, Lange $C$ et al. Patientenversorgung im Großschadens- und Katastrophenfall; Deutscher Ärzteverlag; 2014: 509-515

[6] DIN. 13050:2009-02 (Rettungswesen).

[7] Anlage 5 zur Richtlinie zur Bewältigung von Ereignissen mit einem Massenanfall von Notfallpatienten und Betroffenen (MAN-RL), Bayer. Staatsministerium des Innern, für Bau und Verkehr vom 06.12.2016.

[8] Dittmar MS, Wolf P. Nichtärztliche Vorsichtung beim Massenanfall von Verletzten. Handlungskompetenz lässt innerhalb eines Jahres deutlich nach. Notfall Rettungsmed 2016; 19: $108-114$

[9] Bundeszentrale für politische Bildung: Chemische Kampfstoffe - eine Typologie. Im Internet: http://sicherheitspolitik.bpb.de/ massenvernichtungswaffen/hintergrundtexte-m6/Typologiechemischer-Kampfstoffe, http://sicherheitspolitik.bpb.de/ massenvernichtungswaffen/hintergrundtexte-m6/Chemische-Waffen, Tag des Abrufs 07.11.2017

\section{Bibliografie}

DOI https://doi.org/10.1055/s-0043-119299

retten 2017; 6: 372-381

(c) Georg Thieme Verlag KG, Stuttgart · New York ISSN 2193-2387 
Punkte samneln auf cEFthienede

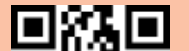

Diese Fortbildungseinheit ist 12 Monate online für die Teilnahme verfügbar.

Sollten Sie Fragen zur Online-Teilnahme haben, finden Sie unter cme.thieme.de/hilfe

eine ausführliche Anleitung. Wir wünschen viel Erfolg beim Beantworten

der Fragen!

Unter https://eref.thieme.de/ZZX92EN oder über den QR-Code kommen Sie direkt zum Artikel zur Eingabe der Antworten.

\section{Frage 1}

Welche Aufgabe gehört nicht zu den Aufgaben eines „ersteintreffenden" Notfallsanitäters/Rettungsassistenten?

A Bestätigung der Funktionsübernahme und Rückmeldung der "Lage auf Sicht“.

B Kennzeichnung mit Weste „Sichtung/Vorsichtung“ und Beginn mit der Sichtung.

C Erkundung und Lagemeldung.

D Abstimmung mit anderen Fachdiensten.

E Organisation der medizinischen Erstversorgung in enger Abstimmung mir dem ersteintreffenden Notarzt.

\section{Frage 2}

Welche Aussage zur Aus- und Fortbildung von Sichtungsteams ist falsch?

A Die Ausbildung von Sichtungsteams soll durch zertifizierte Multiplikatoren erfolgen.

B Die Fortbildung der Sichtungsteams soll durch praktische Übungen erfolgen.

C Die Handlungskompetenz bei der Sichtung kann auch durch Computersimulation erreicht werden.

D Bei der Schulung sollen auch die emotionalen Aspekte berücksichtigt werden.

E Die Kommunikation mit Betroffenen, bzw. Ersthelfern soll eingeübt werden.

\section{Frage 3}

Welche Aussage zum Einsatz eines Sichtungsteams ist richtig?

A Das erste Sichtungsteam wird am Schwerpunkt des Einsatzes eingesetzt.

B Die Mitglieder eines Sichtungsteams splitten sich auf, um schneller vorgehen zu können und somit Zeit zu sparen.

C Die Sichtungskategorie SK IV (blau) ist bei vital bedrohten Patienten zu vergeben.

D Gehfähige sind mit der Sichtungskategorie SK I (grün) zu kennzeichnen.

E Die Dokumentation (Sichtungsliste) ist direkt der Sanitätseinsatzleitung zuzuführen.

\section{Frage 4}

Welche Aussage zur Vergabe von Sichtungskategorien ist falsch?

A Die Sichtungskategorie SK I (rot) ist zu vergeben, wenn ein liegender Patient keinen tastbaren Radialispuls aufweist.

B Die Sichtungskategorie SK II (gelb) ist zu vergeben, wenn bei einem liegenden Patient eine Atmung nicht festgestellt werden kann.

C Die Sichtungskategorie SK III (grün) ist zu vergeben, wenn ein Gehfähiger verletzt ist und kein kritischer Befund festzustellen ist.

D Liegen bei einem Patienten mit dem Leben nicht vereinbare Verletzungen vor, wird eine Todesfeststellung veranlasst.

E Können von einem Patienten einfache Befehle nicht befolgt werden, ist die Sichtungskategorie SK I (rot) zu vergeben.

\section{Frage 5}

Welche Aussage zu Begleitfaktoren bei der Vorsichtung ist falsch?

A Die Anwendung der Vorsichtung wird sehr selten angewendet werden müssen, darin ist eine gewisse Unsicherheit begründet

B Die kurzen Kontaktzeiten zu den Patienten sind ungewohnt.

C Die Konfrontation mit einer Vielzahl von Schwer- Verletzten bzw. Erkrankten erhöht die emotionale Belastung enorm.

D Angehörige bzw. Dritte halten Vor-Sichtungsteams fest, bzw. drohen Gewalt an, wenn der Sichtungsvorgang abgeschlossen ist

E Die Tätigkeit im Vor-Sichtungsteam stellt keine größere Belastung für Einsatzkräfte dar.

\section{Frage 6}

Welche Aussage zum Verdacht auf eine ABC-Lage ist falsch?

A An der Einsatzstelle ist ein Geruch z. B. nach Knoblauch, Senf, Fisch, Heu, Bittermandel oder Chlor festzustellen.

B Gleiche Vergiftungssymptome werden bei mehr als zwei Personen beobachtet.

C Gleiche Vergiftungssymptome werden bei mehr als einer Person beobachtet.

D Der Verdacht auf eine ABC-Lage ist umgehend zu kommunizieren.

E Eine eigene Kontamination ist unbedingt zu vermeiden.

- Weitere Fragen auf der folgenden Seite... 


\section{Punkte sammeln auf CEE.thieme.de}

\section{Frage 7}

Welche Aussage zum Umgang mit "Gehfähigen“ ist richtig?

A Die Vorsichtung in die Sichtungskategorie III (grün) muss bei Erstkontakt zum Patienten durchgeführt werden.

B Die Gehfähigen sollen unbedingt in der Nähe des Schadensortes gesammelt werden.

C Die Differenzierung zwischen Betroffenen und Leichtverletzten darf sinnvollerweise erst nach der Vor-Sichtung der liegenden Verletzten erfolgen.

D Die Nachsichtung nach kritischen Befunden soll nur bei bestimmten Patienten durchgeführt werden.

E Werden bei der Nachsichtung mindestens zwei „kritische Befunde" identifiziert, ist der Patient mindestens in die Sichtungskategorie II (gelb) einzuordnen.

\section{Frage 8}

Welche Aussage zu den Unterschieden bei der Vor- bzw. Nachsichtung zwischen Erwachsenem und Kind ist falsch?

A Die Algorithmen „Vor- bzw. Nachsichtung Kind“ sollen jeweils bis zu einem Patientenalter von 6-8 Jahren angewendet werden.

B Beim Kind wird die kritische obere Atemfrequenz bei 40/min gesehen.

C Beim Kind wird anstelle der Radialispulstastung die Rekap-Zeit an der Stirn überprüft.

D Beim Kind wird die Frage „kann einfache Befehle befolgen“ durch „reagiert adäquat“ ersetzt.

E Eine Herzfrequenz unter 80/min und über 140/min stellt bei der Nachsichtung Kind einen kritischen Befund dar.

\section{Frage 9}

Welche Aussage zur Dokumentation von Sichtungsergebnissen ist falsch?

A Die Nummer der Verletztenanhängekarte ist auf den Dokumentationsbogen zu übertragen.

B Die Sichtungskategorie (SK) ist auf den Dokumentationsbogen zu übertragen.

C Der Patientenname muss auf den Dokumentationsbogen übertragen werden.

D „Patienten“ mit tödlicher Verletzung bekommen keine Verletztenanhängekarte.

E Das Sichtungsergebnis ist an den jeweiligen Abschnittsleiter zu übergeben.

\section{Frage 10}

Welche Aussage zur Anwendung von Sichtungskriterien im Regel-Rettungsdiensteinsatz ist falsch?

A Die Kriterien des Algorithmus Vor-Sichtung können zur objektivierbaren Identifikation von SK I- Patienten angewendet werden.

B Das Kriterium „kann einfache Befehle befolgen“ eignet sich bei erwachsenen Patienten nicht zur Einschätzung im Regel-Rettungsdiensteinsatz.

C Durch die Anwendung der Vor-Sichtungs-Kriterien können begrenzte Ressourcen zielgerichteter eingesetzt werden.

D Bei beteiligten Kindern ist bei dem Kriterium „Atemfrequenz zwischen 10 und 30/min?" der obere Wert auf 40/min anzupassen.

E Bei beteiligten Kindern soll das Kriterium „Radialispuls vorhanden?" durch das Kriterium „Rekap-Zeit Stirn kleiner 3 sec.?“ Ersetzt werden 